\title{
VOLTAGE PROGRAMMABLE DUAL-BAND BANDPASS/BANDSTOP FILTER RESPONSE IN A SINGLE MICRO-ELECTRO-MECHANICAL DEVICE
}

\author{
X. Zou ${ }^{l, *}$, J. Yan ${ }^{l}$ and A. A. Seshia ${ }^{l}$ \\ ${ }^{1}$ Nanoscience Centre, Department of Engineering, University of Cambridge, Cambridge, United Kingdom
}

\begin{abstract}
This paper reports on a switchable multi-band filter response achieved within a single micro-electro-mechanical device. A prototype device fabricated in a SOI process demonstrates a voltage programmable and tunable, dual-band, band-pass/band-stop response. Both analytical and finite element models are introduced in this paper to elucidate the operating principle of the filter and to guide filter design. Voltage programmability of the filter characteristic is demonstrated with the ability to independently tune the centre frequency and bandwidth for each band. A representative measurement shows that the minimum 3 dB-bandwidth (BW) is $155 \mathrm{~Hz}, 140 \mathrm{~Hz}$, and $20 \mathrm{~dB}-\mathrm{BW}$ is $216 \mathrm{~Hz}, 203 \mathrm{~Hz}$ for the upper-band and lower-band center frequencies located at $131.5 \mathrm{kHz}$ and $130.7 \mathrm{kHz}$, respectively.
\end{abstract}

\section{KEYWORDS}

dual-band filter, resonators, voltage programmability.

\section{INTRODUCTION}

Microfabricated silicon resonator-based band-pass filters are being studied for their potential to replace traditional crystal and SAW filters in wireless communication systems [1]. These devices provide the advantages of frequency tunability, relatively small bandwidth and good out-of-band rejection. Band-pass filter topologies usually combine several micro-electro-mechanical (MEM) resonators in series with mechanical and/or electrical coupling elements to construct filter response. However, each device element in the array offers a single pass-band and/or stop-band response only [2]. In this paper, a MEM filter topology is demonstrated which achieves programmable and tunable, dual-band, band-pass/band-stop filter response within a single device. The filter utilizes a self-switching property associated with electrostatic transduction [3] where inverting the polarity of the DC bias on the surrounding electrodes is sufficient to change the response of the device from pass-band to stop-band and vice-versa. As opposed to the results reported in [3], dual-band filter response is achieved within a single device.

\section{FILTER DESIGN AND MODELING}

\section{Design of the filter}

The proposed dual-band filter is based on a new topology comprising two pairs of identical beams connected to a central proof mass at right angles (as shown in Fig. 1). The filter utilizes the first four in-plane modes of this device as shown in Fig. 2 to construct the dual-band filter response. The frequency of each mode may be electrostatically tuned using the electrode configuration shown in Fig. 3. Two adjacent electrodes are used as signal input ports (Port 1 and Port 4 shown in Fig. 3) and the other two adjacent electrodes are used as signal output ports (Port 2 and Port 3 shown in Fig. 3).

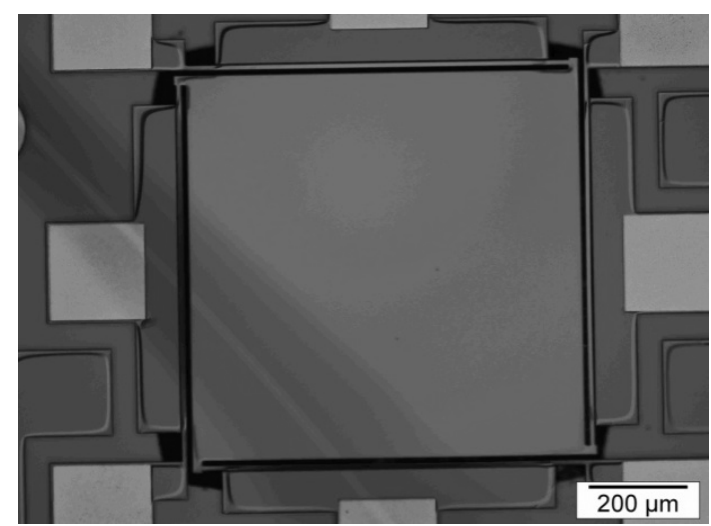

Figure 1. Optical micrograph of the MEM dual-band filter

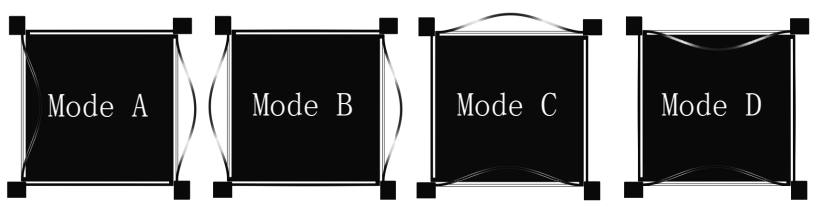

Figure 2. First four in-plane mode shapes used to construct the dual-band filter (COMSOL 3.5).

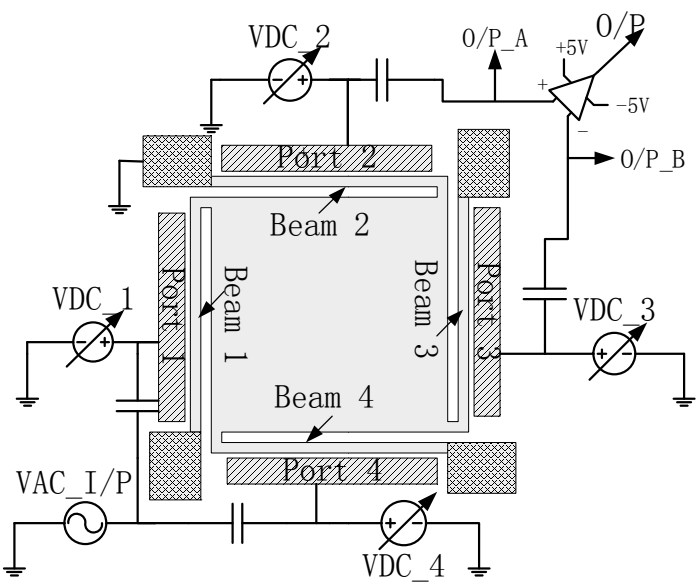

Figure 3. Schematic view of dual-band tunable filter with tuning circuit.

The device can be operated in a number of different modes depending on the polarity of the voltages applied to the surrounding electrodes. The device can switch between single-band and dual-band operation modes, as 
well as between a band-pass and band-stop response. This programmability of filter response is achieved by changing the polarity of the DC voltage applied to each port thereby changing the sign of the associated electromechanical transduction parameter. A single-band response is achieved by setting the associated transduction parameter for one of the two coupled modes to zero by switching off the DC bias voltage on the corresponding electrodes. Table 1 summarizes the programming characteristic of the filter for the topology presented here.

Table 1. Programmable operation of the dual-band filter

\begin{tabular}{|l|c|c|c|c|}
\hline \multirow{2}{*}{ Filter O/P } & \multicolumn{4}{|c|}{ Polarity of DC Voltage } \\
\cline { 2 - 5 } & $V D C \_1$ & $V D C \_2$ & $V D C \_3$ & $V D C \_4$ \\
\hline Single Band-Pass & 0 & $-/+$ & 0 & $+/-$ \\
\hline Single Band-Stop & 0 & $+/-$ & 0 & $+/-$ \\
\hline Single Band-Pass & $+/-$ & 0 & $+/-$ & 0 \\
\hline Single Band-Stop & $+/-$ & 0 & $-/+$ & 0 \\
\hline Dual B-P/B-S & $+/-$ & $-/+$ & $-/+$ & $+/-$ \\
\hline Dual B-P/B-P & $+/-$ & $-/+$ & $+/-$ & $+/-$ \\
\hline Dual B-S/B-S & $+/-$ & $+/-$ & $-/+$ & $+/-$ \\
\hline Dual B-S/B-P & $+/-$ & $+/-$ & $+/-$ & $+/-$ \\
\hline
\end{tabular}

\section{Analytical modeling}

Since the filter response is determined by the four modes of this device (shown in Figure 2), an analytical model is useful to interpret the operating principle of the filter and to provide the basis for further design extensions. This device can be modeled as a weakly coupled spring-mass system with 4 degrees of freedom (DOF) (see Fig. 4(a)) in the operating band of interest. Each of the four beams is modeled as a 1-DOF spring-mass system where the stiffness and effective mass is estimated by approximating each beam to the clamped-clamped (CC) boundary condition. The central proof mass that couples the four beams may, in turn, be modeled as a weak variable coupling spring $\left(\mathrm{k}_{\mathrm{c}, \mathrm{i}}\right)$, as it simply connects the four beams at the points of minimum kinetic energy resulting in a coupling spring with a dynamic stiffness whose value is defined by the pairs of beams under consideration. This model can be further simplified (Fig.4 (b)) by decoupling the observed response along the two axes of vibration. Two of the four modes originate from coupled resonant vibrations along the $x$ axis while the remaining two modes originate from coupled resonant vibrations along the $y$ axis as shown. Further, the simplified model ignores the coupling (although very weak coupling exists in practice) between the $x$ and $y$ directions.

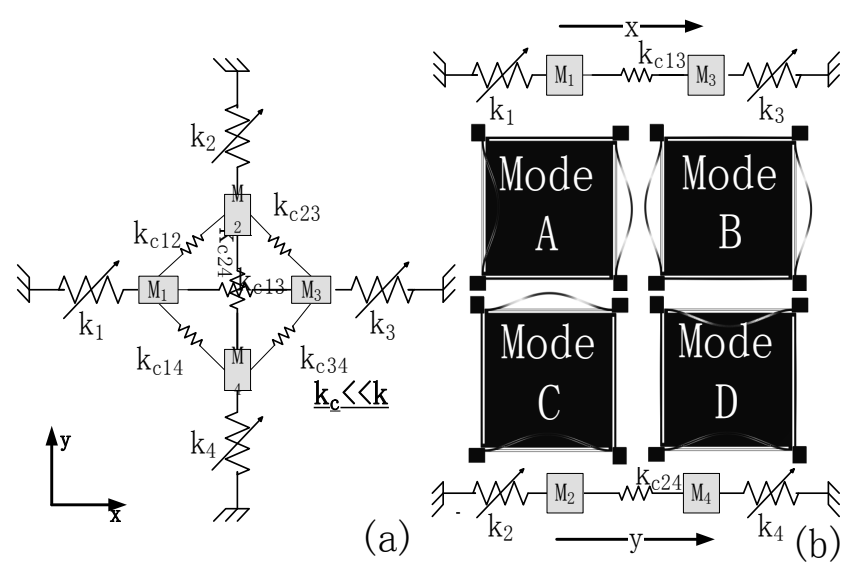

Figure 4. (a)Analytical model with 4 DOF (b) Simplified analytical model by decoupling response along $x$ and $y$-axes

In this case, the frequency difference of the two $x$ (or $y$ ) modes can be estimated by [2]:

$$
\omega_{B}^{2}-\omega_{A}^{2} \approx \frac{\sqrt{\left(k_{1}-k_{3}\right)^{2}+4 k_{c 13}^{2}}}{M_{e f f}}, \omega_{D}^{2}-\omega_{C}^{2} \approx \frac{\sqrt{\left(k_{2}-k_{4}\right)^{2}+4 k_{c 24}^{2}}}{M_{e f f}}
$$

For the dual-band filter design, the two modes in the $x$-axis construct one pass/stop band and two other modes in the $y$-axis construct the other pass/stop band. Thus, Eq. (1) also estimates the bandwidth of each pass-band or stop-band. The sideband frequencies of each pass-band or stop-band can be tuned independently by the electrostatic spring softening effect and the center frequency of the two bands is set by beam dimensions.

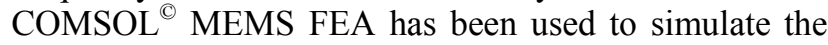
center frequency of a given band as a function of the dimensions of a given pair of beams. This has been compared to the analytical model and good agreement is found (Fig.5).

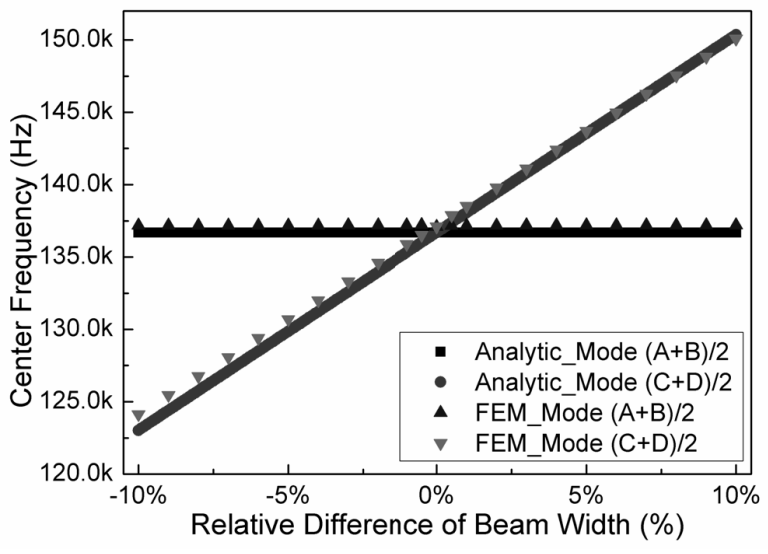

Figure 5. Comparison of analytical modeling and FEM simulation of the dual-band center frequency as a function of the width of beams 2 and 4 . 


\section{FEM model of the filter}

Although the simplified analytical model can predict the overall response of the filter, an FEM model and associated simulations are also necessary to study the characteristics of the filter in detail. All FEM modeling and simulation in this section are based on models developed in COMSOL $^{\circ}$ MEMS FEA software. As discussed in the previous section, the center frequency of the two bands can be set by beam dimensions. However, due to the weak coupling between the modes in $x$ and $y$ directions (which is ignored in the reduced analytic model), the center frequencies of the two bands cannot be set very close to each other. To study the cross-axis coupling effect on the center frequency of the two bands of the filter, the simulation of the frequency of the four modes of interest as a function of the width of one pair of beams is conducted and the results are shown in Fig. 6 .

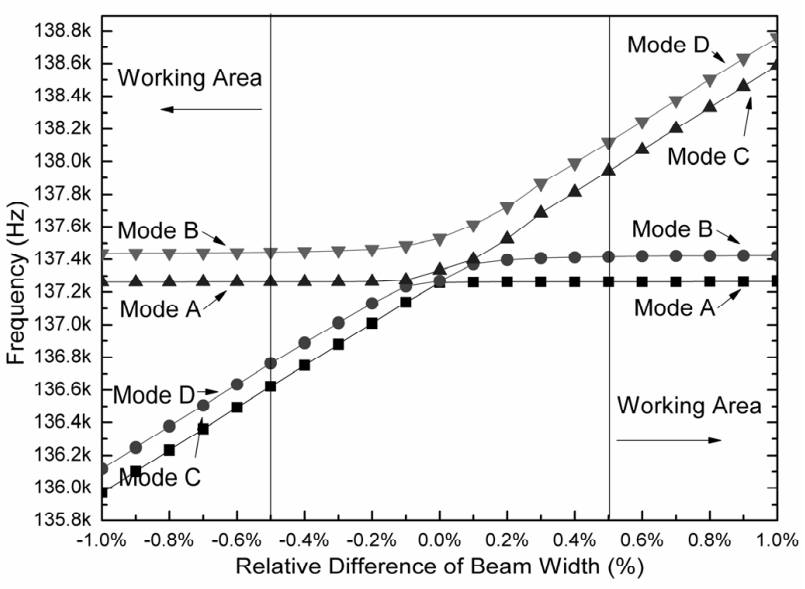

Figure 6: FEM simulation of the frequencies of the four modes as a function of the width of beams 2 and 4 .

In this simulation, the width of two beams in $x$ direction (beams 1 and 3) were constant and the width of two beams in $y$ direction (beams 2 and 4) were changed from $-1 \%$ to $1 \%$ with respect to that of the beams 1 and 3 . The simulation results indicate that the center frequency of each band can be set independently by the beam widths only if the relative difference between the beams in $x$ and $y$ directions is more than $\pm 0.5 \%$ or the mode shape will distort and center frequencies will shift due to the cross-axis modal coupling. In other words, designing the beam dimensions appropriately should ensure the filter operates in the working zone shown in Figure 6.

For a correctly designed filter which operates in the working zone discussed above, the bandwidth of each band can be independently tuned by changing the stiffness of one of the beams of the pair associated with each mode. This tuning can also be dynamically achieved by the electrostatic spring softening effect in practice. Simulations show the effects of frequency tuning by varying the width of one beam slightly to change the effective stiffness of the beam. Figure 7 (a) shows the upper-band tuned by the width of beam 2 and Fig. 7(b) shows the lower-band tuned by changing the width of beam 1. The minimum bandwidth of each band is determined by the coupling stiffness, $\mathrm{k}_{\mathrm{c}}$, as shown in Eq. 1. However, this stiffness cannot be easily derived from the device topology and dimensions and must be estimated from simulation.

Finally, the programmability of the dual-band filter characteristic as shown in Table 1 was simulated as well. Figure 8 shows the programmable band-pass/band-pass, band-stop/band-stop and band-pass/band-stop dual-band filter responses obtained through simulation. The simulation results agree with the overall experimentally observed filter response. The center frequencies of the fabricated devices are slightly lower than the simulation results potentially due to the fabrication tolerances associated with the beam widths.

\section{EXPERIMENTAL RESULTS}

Experimental validation of the device concept is demonstrated based on prototypes fabricated in a SOI foundry process. Figure 9 shows that the bandwidth for each band of the dual-band filter can be independently tuned by varying the magnitude of the DC bias voltages applied on the associated electrodes. The results show that the minimum 3dB-bandwidth is $155 \mathrm{~Hz}, 20$ $\mathrm{dB}$-bandwidth is $216 \mathrm{~Hz}$ and the ripple is $13.2 \mathrm{~dB}$ for the upper-band with center frequency between $131.775 \mathrm{kHz}$ and $131.477 \mathrm{kHz}$. The minimum $3 \mathrm{~dB}-\mathrm{BW}$ is $140 \mathrm{~Hz}, 20$ $\mathrm{dB}-\mathrm{BW}$ is $203 \mathrm{~Hz}$ and ripple is $13.1 \mathrm{~dB}$ for the lower-band with center frequency between $130.452 \mathrm{kHz}$ and 130.085 $\mathrm{kHz}$. The ratio of minimum $3-\mathrm{dB}$ bandwidth to center frequency is approximately $0.11 \%$ and the ratio of the minimum $20-\mathrm{dB}$ bandwidth to center frequency is approximately $0.17 \%$.

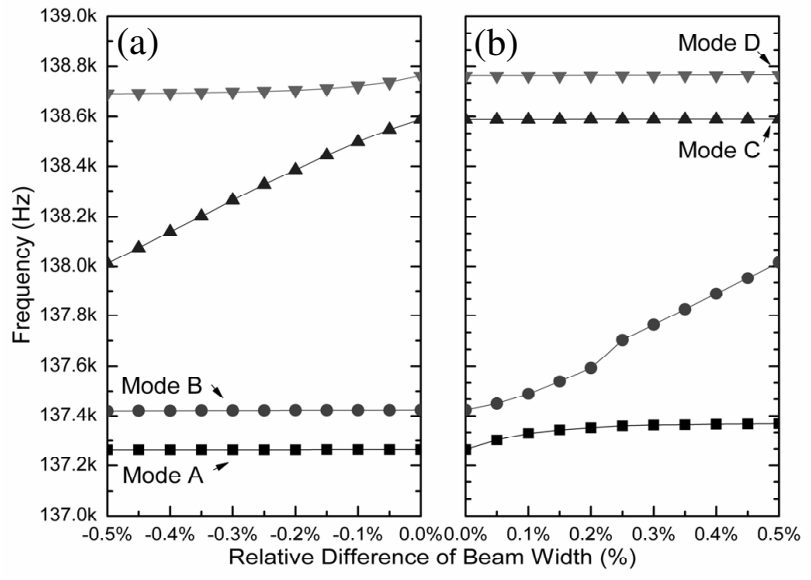

Figure 7. FEM simulation demonstrating independent tuning of the upper band (a) and lower band (b)of the filter.

Figure 10 demonstrates the voltage programmability of the dual-band filter response. The results indicates that the dual-band filter can be programmed as a single band-pass/band-stop filter or as a dual-band filter with two pass-bands, two stop-bands or one pass-band and one stop-band with all bands of the same approximate bandwidth and each band-width can be independently 
tuned as well.
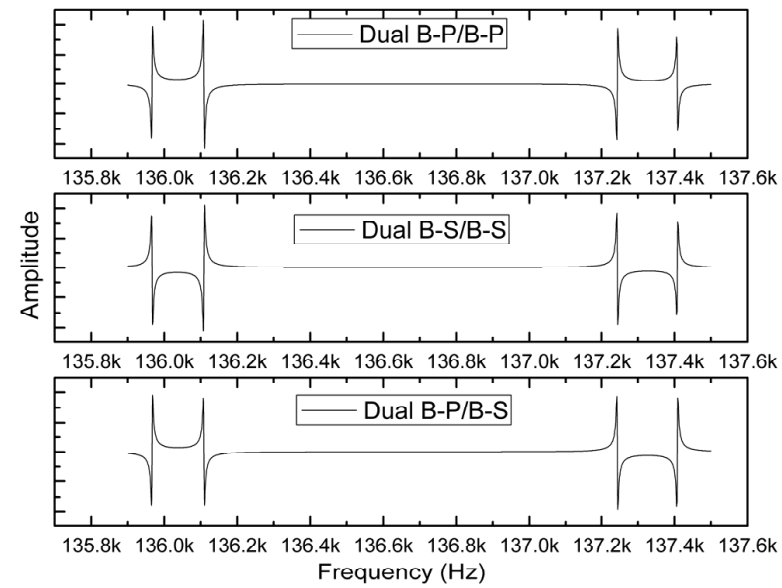

Figure 8. Simulation of the programmable dual-band filter response.

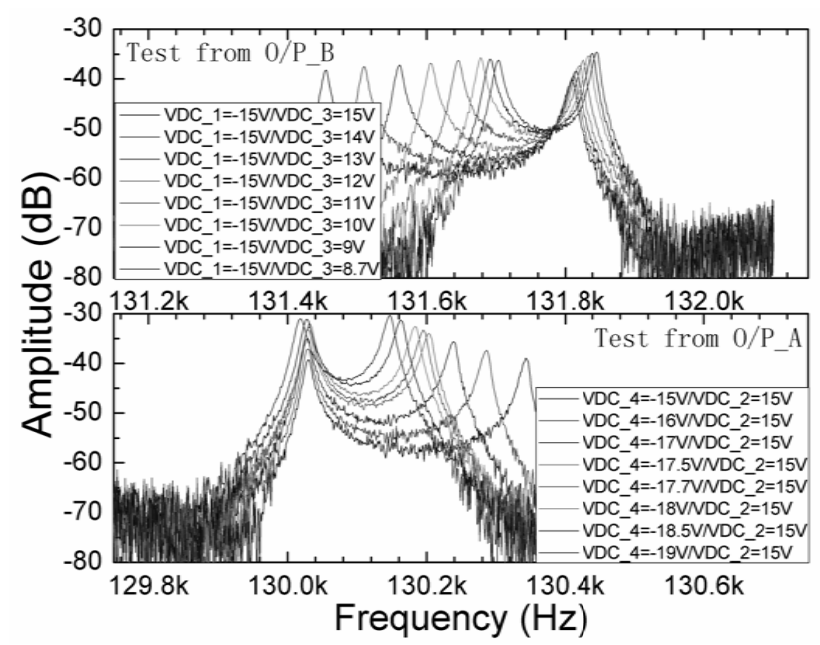

Figure 9. Tuning the two bands of the dual-band filter independently by electrostatic spring softening.

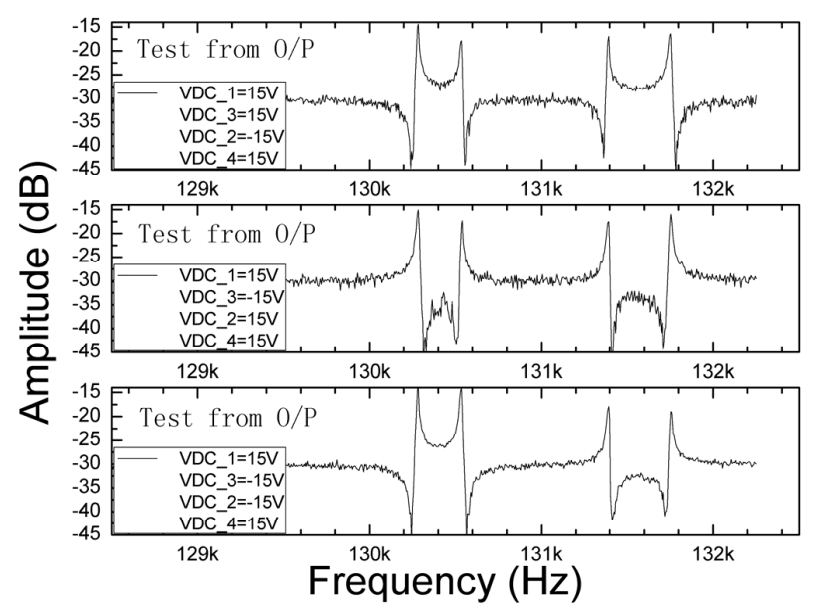

Figure 10. Experimentally observed voltage programmable dual-band filter response.

\section{CONCLUSIONS AND FUTURE WORK}

This paper introduced and demonstrated a voltage programmable dual-band filter response within a single MEM device. The analytical models and FEA results exhibited good agreement with experiment. This new topology demonstrates the design flexibility associated with MEM resonator based filter design, leveraging electrostatic tuning and the programmability of the electrostatic transduction parameter together with selective and directional modal coupling to provide filter functionality not possible using more conventional technologies. This concept can be extended to filters operating in the VHF - UHF through appropriate technology scaling [1] as well as to achieving a multi-band filter response based on the selective coupling of a large number of interconnected elements within a single device.

\section{REFERENCES}

[1] C. T. C. Nguyen, "Vibrating RF MEMS overview: Applications to wireless communications," in Micromachining and Microfabrication Process Technology X. vol. 5715, 2005, pp. 11-25.

[2] J. Yan et al., "Internal electrical and mechanical phase inversion for coupled resonator-array MEMS filters," Sensors and Actuators a-Physical, vol. 158, pp. 18-29, Mar 2010.

[3] $\mathrm{S}-\mathrm{S} \mathrm{Li}$ et al., "Self-switching vibrating micromechanical filter bank," in Joint IEEE Int. Frequency Control/ Precision Time \& Time Interval Symposium, Vancouver, Canada, 2005, pp. 135-141.

\section{CONTACT}

* X. ZOU, tel: +44-122-376-0304; xz280@ cam.ac.uk 